\title{
Basal articulation of the palps and ovigers in Antarctic Colossendeis (Pycnogonida; Colossendeidae)
}

\author{
Esperanza Cano-Sánchez and Pablo J. López-González
}

\begin{abstract}
Selected sea spider specimens of Antarctic Colossendeis species collected during the Italica XIX cruise and the Polarstern cruise ANT XXIII/8 were examined to provide new information about the external and internal anatomy of the basal parts of the palps and ovigers. The presence and insertion of the muscle bands, as well as the arthrodial membrane are illustrated and discussed. The results obtained in this study show that the basal parts of the palps and ovigers have a similar internal structure. This is in agreement with the currently established 10-articled status for the ovigers (the basal element is not considered an article). Despite the currently established 10-articled status for the palps, our results suggest that the palp should be considered as being 9-articled.
\end{abstract}

Keywords: Sea spiders, Basal articulation, Scanning electron microscopy, Light microscopy, Musculature

\section{Background}

The pycnogonids or sea spiders are a monophyletic group; they are defined by autapomorphies such as the prominent proboscis, an extremely reduced abdomen, and the presence of a ventral pair of appendages (ovigers) on the cephalon [1]. This group contains more than 1300 described species [2], of which more than 260 are Antarctic and Subantarctic species [3].

Two suborders, Stiripasterida and Eupantopodida, are currently considered in the Pantopoda, the only extant Pycnogonida order [4]. Some contributions based on molecular data suggested that the Austrodecidae (the only family included in Stiripasterida) is the basal-most group, being the sister group of the remaining extant families (all them in Eupantopodida) [2, 5]. In this last clade, including $95 \%$ of the extant pycnogonids species, the families Pycnogonidae and Colossendeidae are the basal-most groupings. In Austrodecidae, palp variation ranges from 5- to 9-articled, ovigers being absent or up to 10-articled. In Pycnogonidae, palps are absent,

\footnotetext{
*Correspondence: ecano@us.es

Biodiversidad y Ecología de Invertebrados Marinos, Departamento de Zoología, Facultad de Biología, Universidad de Sevilla, Avda. Reina Mercedes 6, 41012 Seville, Spain
}

but ovigers are only present in males, 4- to 9-articled. Colossendeidae have been characterized as having 9- to 10-articled palps and 10-articled ovigers, both structures being present in male and female sexual forms [4]. Palps are considered to be the homologues of pedipalps in arachnids [6,7], while ovigers are interpreted as modified legs [8], having primarily a cleaning function and assuming secondarily the transport of egg-masses, a task carried out in pycnogonids by the male individuals [4].

Traditionally, the classification of pycnogonid families has been based on the presence or absence of cephalic appendages (chelifores, palps, and ovigers) in adults, and gradual article reduction has been suggested to have occurred during the group's evolution [4, 6, 9-11]. However, reduction or loss of these appendages represents a polyphyletic condition in Pycnogonida [2, 4, 6]. Chelifores, palps, and ovigers, also show a wide range of variation in number of articles and other external features, and this structural and ornamental variability seems to be valid even within some genera and among ontogenetic stages [6].

Colossendeidae has a predominantly Antarctic and Subantarctic distribution [2], with three genera present in this area: Colossendeis (the majority of the species), Decolopoda and Dodecolopoda [12]. This family is 
characterized by the following morphological characters in adults: (1) four (Colossendeis) to six (Decolopoda and Dodelocopoda) trunk segments; (2) ovigers 10-articulated; (3) compound oviger spines in fields or rows on a strigilis; (4) gonopores on all legs; (5) palps 9- or 10-articulated [4]. The number of palpal articles (9- or 10-) is now accepted in accordance with Child's [12] considerations. Before this author's work, the accepted condition was 8- or 9-articled, as the first articles were considered to be basal processes (see $[8,13-18]$ ), although some earlier authors believed it was 10-articled [19-21].

Cano and López-Gonzalez [22] discussed the 10- or 11articled nature of the ovigers in the Colossendeis species based on their similarities with the basal parts of palps. If a 10- articled palp is considered, an 11- articled oviger should not be rejected; both considerations seem to be linked. The controversy about the number of articles making up the ovigers and palps is an open question.

This paper attempts to provide some information concerning the presence and placement of muscle bands in the basal parts of palps and ovigers observed in the Antarctic and Subantarctic species of the genus Colossendeis, which were subjected to an internal SEM and light microscopy study.

\section{Methods}

The material examined for this study was identified as Colossendeis megalonyx Hoek, 1881, C. australis Hodgson, 1907, C. tortipalpis Gordon, 1932, C. scotti Calman, 1915, and C. glacialis Hodgson, 1907.

The material studied was collected on the R/V Italica cruise, XIX Spedizione (Victoria Land Transect cruise, from 3 February to 4 March 2004) and R/V Polarstern cruises, ANT XXIII/8 (from 23 November 2006 to 30 January 2007) to the Ross Sea and to Elephant Island. The specimens were sorted, fixed in $10 \%$ buffered formalin in seawater and then transferred to $70 \%$ ethanol.

The specimens collected were identified by the authors. The literature used for the identification of the specimens mainly includes the most important works carried out in the last century on Antarctic pycnogonid fauna [12, 14$18,21,23-25]$.

Fragments of selected specimens were examined in order to study three specific areas: (1) the basal palp articles; (2) the basal oviger articles; and (3) the first or second trunk segment with lateral processes and the first basal articles of the first or second leg. These fragments were dehydrated in butanol [26], and embedded in paraffin. Precise cuts were carried out using a microtome, where longitudinal sections about $10 \mu \mathrm{m}$ thick were removed from the paraffin blocks (including the pycnogonid fragments) until about half of the appendage under study (palp, oviger, or lateral process plus coxae) was visible, which made observation of the gross internal anatomy possible. The remaining fragments included in the paraffin blocks were deparaffinized in xylol, and prepared for the SEM study, critical point-dried, mounted on stubs, coated with gold-palladium and observed with a Philips XL30 SEM. Other fragments were stained in an aqueous solution of fuchsin basic (Panreac $\odot$ cod. 251332), dehydrated in a series of ethylic alcohol, permanent mounted in DPX (Panreac@ cod. 255254.1610), and observed with light microscopy.

In this text and figures, the apparent first basal article of the palp and oviger are named $p b p$ (palp's basal process), or $o b p$ (oviger's basal process), while the following articles distally will be named as $p 1, p 2, p 3, \ldots$ (for palp articles) and $o 1, o 2, o 3, \ldots$ (for oviger articles). The lateral process on the trunk will be referred to with the abbreviation $l p$, while the coxae will be named as $c 1, c 2$, and $c 3$.

\section{Results}

The cuticular gross structure of palpal and ovigeral basal parts examined for the present study is similar. No differences have been observed among the Colossendeis species examined in this study.

Articles of a limb (as those found in palps, ovigers or walking legs) are here considered as elements having arthrodial membranes between consecutive articles, as well as between a trunk segment and the first article of a limb. Articles are articulated by an extrinsic or intrinsic set of muscle bands. Extrinsic musculature spans from the trunk into the limb, whereas the intrinsic ones represent muscle bands that interconnect limb articles.

In the following paragraphs the observed structure of the basal palpal and ovigeral parts in the studied material will be described:

\section{Basal palp articles and processes}

Externally, although the short basal process palp ( $p b p)$ has a swollen appearance (see Fig. 1) and a shallow delimitation between the surface of the trunk and those of the $p b p$ (Fig. 1d), there is no arthrodial membrane, joint or discontinuity, as observed between it and the first palpal article ( $p 1)$.

Internally, there are some sets of longitudinal extrinsic muscle bands $(e m b)$ which originate on the lip of the opening of the cephalon and proximal areas of the $p b p$, and they insert into the distal part of $p 1$. These emb cross the $p b p$ and $p 1$, but we have not detected muscle bands from the cephalon to the $p b p$ (extrinsic in that case) suggesting a possible article condition for the here-named pbp (Figs. 2a, 3b, c).

In the cuticular gross structure we detected distinct differences between the $p b p$ and $p 1$ (Fig. 2b), corresponding to the presence of an arthrodial membrane. Otherwise, 
there is a structural continuity between the cephalon and the $p b p$ (see Fig. 2a), as well as between the $p b p$ and the closely placed oviger basal process $(o b p)$ (Fig. 2c).

\section{Basal oviger articles and processes}

As in the previous case, externally, the oviger basal process (herein as $o b p$ ) has a similar aspect to the following three short basal oviger articles (o1,o2, and $o 3$ in Fig. 1). There is an indistinct delimitation between the surface of the trunk and those of the obp (Fig. 1d), there is no arthrodial membrane, joint or discontinuity, as observed between the basal process and the following article (o1).

Internally, as occurred in the palp, there are some sets of longitudinal extrinsic muscle bands $(\mathrm{emb})$ which originate on the proximal areas of the $o b p$, reaching to the proximal part of $o 1$; but no muscle bands from the cephalon to the $o b p$ were detected (Figs. 2d, 3a, b). An intrinsic band of muscles is visible from the half proximal part of $o 1$ to the proximal part of $o 2$ (Figs. 2d, 3a). A similar situation occurs in the intrinsic muscle bands between the following pairs of articles (o2-o3, o3-o4) (Fig. 2d).

The cuticular gross structure between cephalon, $o b p$ and $o 1$ is similar to that described for the palp (Fig. 2c).

\section{Discussion}

In general, it is accepted that both a reduction in the number of articles in the appendages or the final atrophy of the appendage correspond to derived conditions (or derived character state) (see for additional details $[4,6$, $8,10,11,27])$. In Pycnogonida, palps seem to have their correspondence with pedipalps in other Chelicerata [6, 7], while ovigers are often interpreted as modified walking legs, primarily based on fossil evidence [28].

In Colossendeis the maximum number of articles ( 9 or 10) of the palp has long been discussed. A 10-articled condition was considered by Hoek [19], Wilson [20], and Hodgson [21], while in subsequent papers this appendage was described as 9-articled [8,13-18]. This discrepancy depended on whether or not the basal part was considered as an article.

Currently a 10-articled condition of the palp is accepted for Colossendeis species (except for C. wilsoni which is 9 -articled). This interpretation was again introduced by Child [12]. For this author the suture line between the lateral extension of the cephalon and the first palp article is not always clearly marked, but close examination almost always reveals a separation line. For this reason, one additional short basal palp article must be considered, and thus the maximum number of articles passed from nine to ten (or eight to nine in the case of $C$. wilsoni).

Hence, if this basal element were to be considered an article, it should also retain a set of extrinsic muscle bands which originate on the lip of the opening of the cephalon and insert into the distal part of this basal element. However, our results show the opposite. There is no arthrodial membrane between the cephalic area and this basal element (Fig. 2c); and, furthermore, we have not observed any set of extrinsic muscle bands from the cephalic area to this supposed article (Figs. 2a, 3c). Thus, the basal part cannot be considered an article, and a 9-articled (8-for C. wilsoni) condition is stated here for the palps.

In the case of the oviger structure, a 10-articled condition (plus a terminal claw) is accepted for Colossendeidae species, but the ovigers have an indistinct suture line between the cephalon and the basal part of the ovigers (see [22]: Fig. 14; and Fig. 1 in this paper). Cano and López-González [22] discussed the presence of four short oviger basal articles or an oviger-bearing process followed by three short articles, as is generally accepted in the literature. These authors considered the possibility of an 11-articled condition in Colossendeis ovigers by including this basal part instead of the 10-articled condition described in previous literature. This supposition was reinforced by the presence in some species of a similar ventral ornamentation on the basal part of the oviger and the following oviger articles (see [22]: Fig. 14b), and the similar appearance of the basal part of the palp. As occurred above in the basal part of the palp, in the basal part of the ovigers there is neither set of extrinsic muscle bands nor an arthrodial membrane between the cephalic area and this basal element (Figs. 2d, 3a). Thus, this basal part should no longer be included in the article count.

Sets of extrinsic muscle bands arising from the cephalon and reaching the proximal part of the first palp and oviger articles were observed in Nymphon rubrum Hodge

\footnotetext{
(See figure on next page.)

Fig. 1 External view of Colossendeis specimens: a drawing of the general ventral view of a specimen to locate insertion of the different appendages. $\mathbf{b}$ detail from a, showing basal parts of palps and ovigers. c ventral view of Colossendeis megalonyx showing basal parts of palps and ovigers and arthrodial membranes (black arrows). d SEM photograph of Colossendeis megalonyx, ventro-lateral view of basal part of right palp and oviger (white arrows indicate limit between cephalic area and basal process of palp, discontinuous arrows indicate limit between cephalic area and basal process of oviger). e ventral view of C. tortipalpis showing basal parts of palps and ovigers and arthrodial membranes (black arrows). $\mathbf{f}$ ventral view of C. australis showing basal parts of palps and ovigers and arthrodial membranes (black arrows). Ip lateral process, 01-04 first to fourth oviger article, obp oviger's basal process, $p$ palp, p1 first palp article, $p 2$ second palp article, pbp palp's basal process, pr proboscis, tr trunk
} 

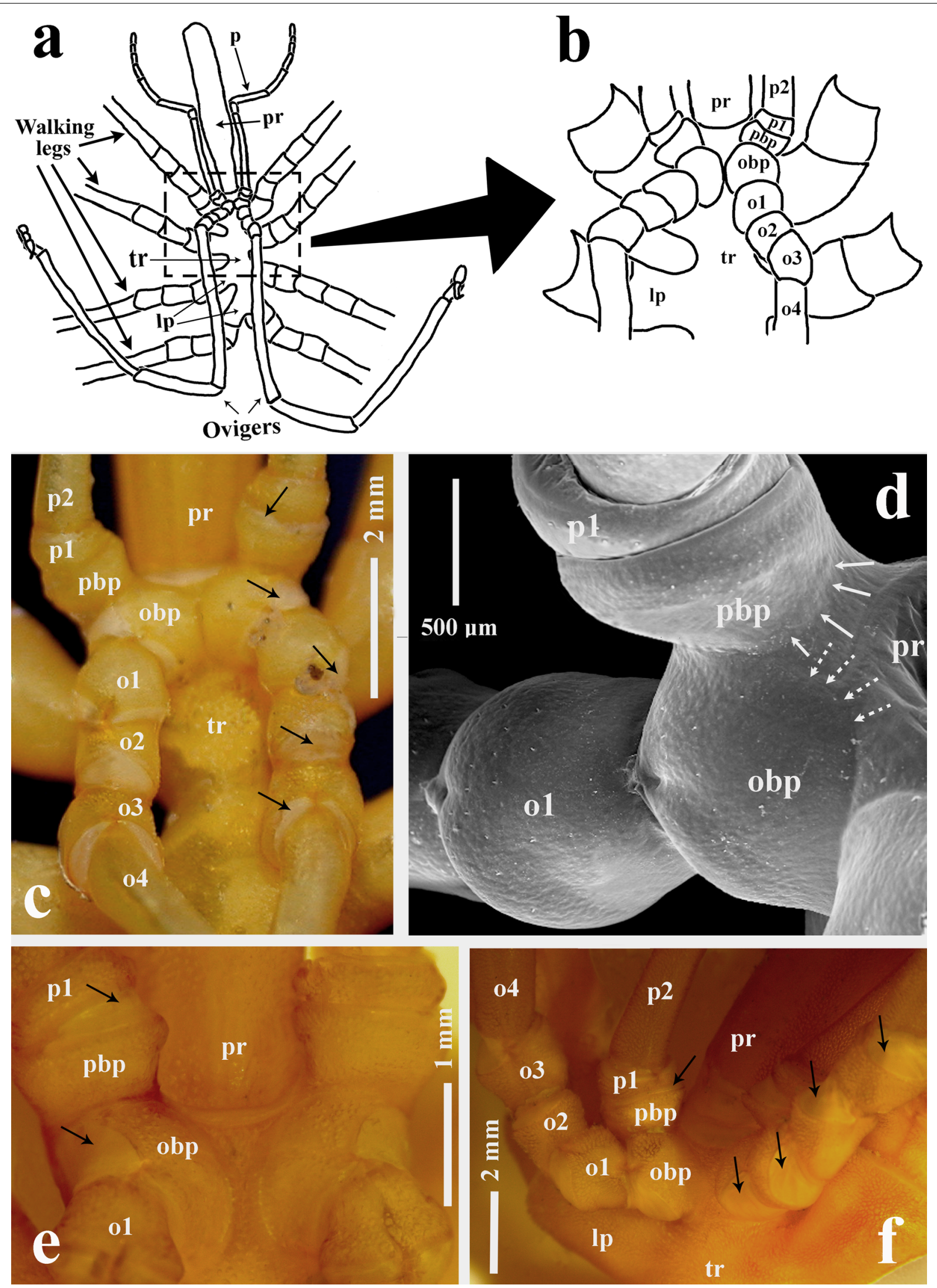


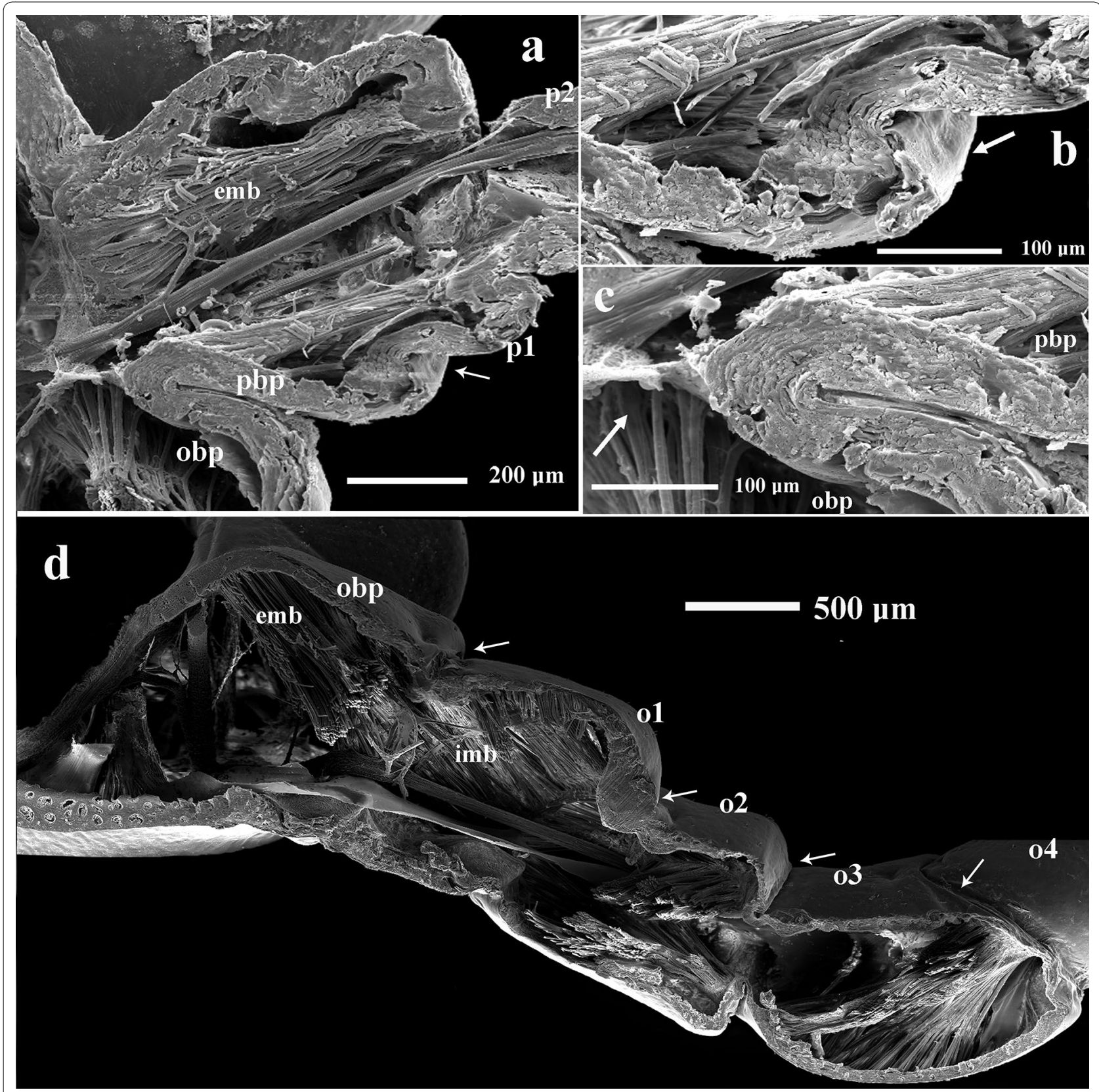

Fig. 2 SEM internal view of Colossendeis megalonyx. a ventral view of left palp, showing sets of muscle bands from the lip of the opening of the cephalic area to the distal part of first article and arthrodial membrane (white arrow). $\mathbf{b}$ detail from a, showing the articulation between the basal process of palp and first article. $\mathbf{c}$ detail from a, showing basal area between cephalic area, the basal process of palp and the basal process of the oviger (arrow indicate insertion of muscle bands of oviger on the opening of the cephalic area). $\mathbf{d}$ posterior view of left oviger, showing extrinsic and intrinsic muscle bands in each article, and arthrodial membranes (white arrow). emb extrinsic muscle bands, imb intrinsic muscle bands, 01-04, first to fourth oviger article; obp oviger's basal process, $p 1$ first palp article, $p 2$ second palp article, $p b p$ palp's basal process

[29]. A similar situation is observed in the Colossendeis species here examined (Figs. 2d, 3a, b); no arthrodial membranes were present, and the gross cuticle structure is similar in the areas between palp and oviger basal processes (Fig. 2c).
Indeed, the situation observed in $p b p$ with respect to $p 1$, and that existing between $o b p$ and $o 1$ is similar to that observed between the lateral processes of the trunk and coxa 1. Schram and Hedgpeth [30] described the sets of muscle bands originating from the lip of the opening 


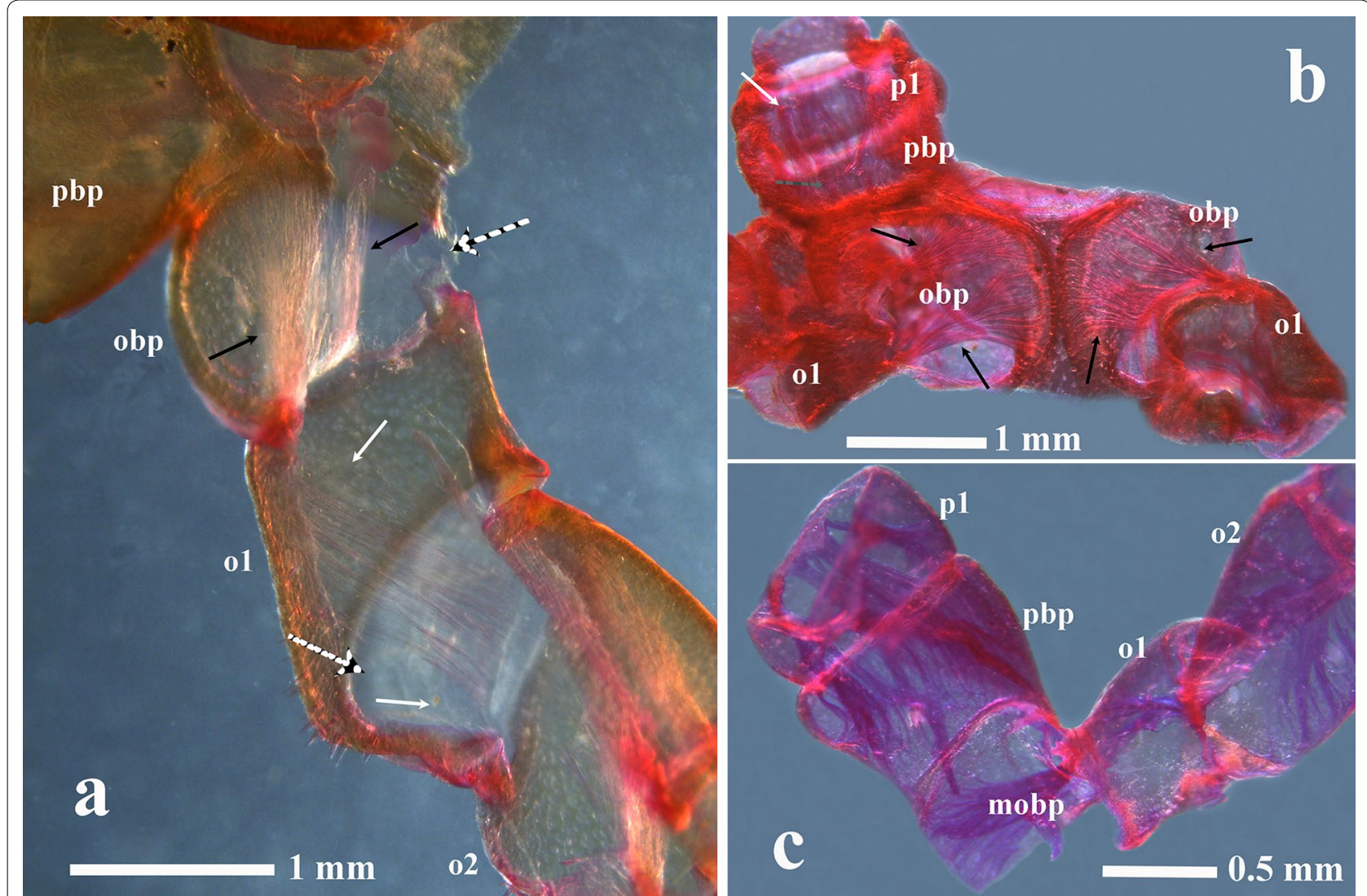

Fig. 3 Light Microscopic longitudinal section of basal parts of palps and ovigers. a dorso-posterior vision of right oviger of Colossendeis scotti, showing extrinsic muscle bands from the opening of the cephalic area to the proximal part of first article (black arrow), intrinsic muscle bands from lateral part of first article to proximal part of the next article (white arrow) and arthrodial membrane (discontinuous white arrow). b ventral view of oviger and left palp of Colossendeis tortipalpis, showing extrinsic muscle bands from the lip of the opening of the cephalic area to the distal part of first palp article (white arrow) and two sets of extrinsic muscle bands from the opening of the cephalic area to the proximal part of first oviger article (black arrow). c posterior view of left palp of Colossendeis megalonyx, showing extrinsic muscle bands from the lip of the opening of the cephalon to the distal part of first article. c1 coxa 1, Ip lateral process, mobp set of muscular band of oviger's basal process, 01-02 first to second oviger article, obp oviger's basal process, $p 1$ first palp article, pbp palp's basal process

of the lateral processes in the trunk cavity to the dorsal and ventral rims of what is considered the first article of the walking legs (coxa 1), using the anatomy of Colossendeis australis as a model (see [30]: Fig. 2a, b). In the present study, the area between the lateral process and coxa 1 has been also examined by SEM (Fig. 4a) and light microscopy (Fig. 4c). We corroborate the presence of the set of muscle described by Schram and Hedgpeth [30]. We detected the presence of an arthrodial membrane between the lateral processes and coxa 1 (Fig. 4b) but not in the base of the lateral processes, where a continuity in layers is observed (Fig. 4a).

These results suggest: (1) the 9-articled condition of the palp of Colossendeis species (8-articled in C. wilsoni), the previously considered first article being a basal process $(p b p)$ followed by nine articles $(p 1, p 2, p 3, \ldots)$; and (2) the generally accepted 10 -articled (plus terminal claw) condition of the ovigers, which have a basal process $(o b p)$ followed by three short articles $(o 1, o 2, o 3)$. As the external condition is similar for the species of the other two colossendeid genera examined (Decolopoda and Dodecolopoda) (Additional file 1: SM. 1), the interpretation given for Colossendeis could be also applicable at least to the Antarctic Colossendeidae.

Wilson ([20]:242) considered the ovigers as "accessory legs 11-(10?) jointed" by adding the terminal claw as a possible eleventh article (see [20]: 246 when describing C. macerrima). For other authors this claw was simply a transformed spine [e.g. 11]. Currently, this terminal claw is not considered an article, and the assumed 10-articled (plus terminal claw) status is considered a derived condition, assuming that the 11-articled oviger of the Devonian fossil Palaeoisopus problematicus Broili is the plesiomorphic state in the group [6, 8, 10, 11, 27, 28]. 


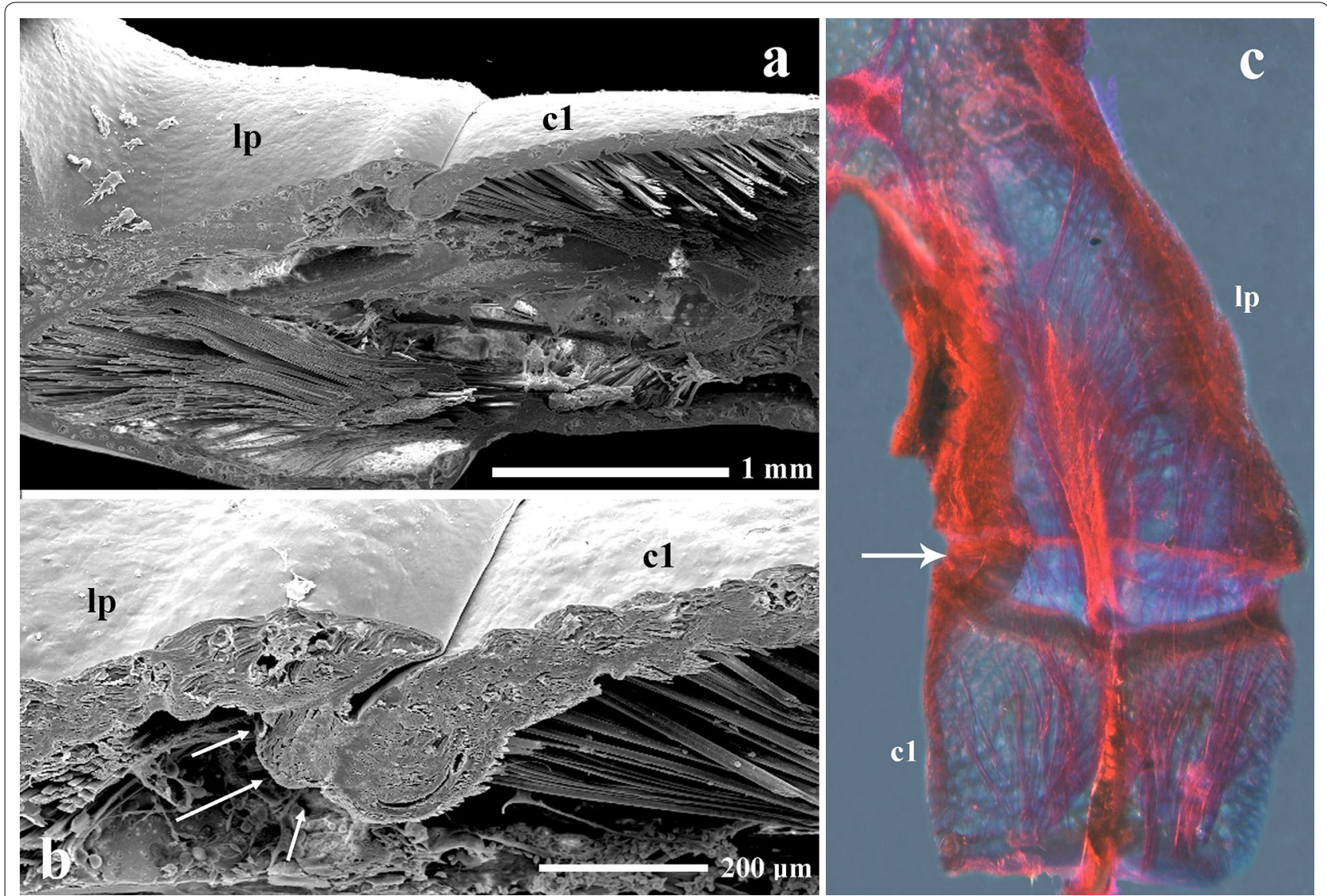

Fig. 4 Internal view of lateral process and coxa 1 of walking leg. a SEM anterior view of lateral process of second segment and coxa 1 of second walking leg of Colossendeis megalonyx, showing sets of muscle bands from the lip of the opening of lateral processes with the trunk cavity to the proximal area of the coxa 1. b detail from $\mathbf{a}$, arthrodial membrane between the lateral processes and the coxa 1 (arrows indicate the arthrodial membrane). c Light microscopic dorsal view of left lateral process of first segment and coxa 1 of first walking leg of Colossendeis tortipalpis, showing sets of muscle bands from the lip of the opening of lateral processes with the trunk cavity to the proximal area of the coxa 1 (arrows indicate the arthrodial membrane). $c 1$ coxa1, Ip lateral process

Both palpal and ovigeral basal parts seems to have undergone similar patterns of evolution. According to the described palpal and ovigeral article reduction in the three basal-most extant families in Pycnogonida: Austrodecidae, Pycnogonidae, and Colossendeidae [2, 5], the Colossendeiidae retain the primitive form of palp and oviger ([4]: 305), with similar expression in males and females.

\section{Conclusions}

The structure of the basal areas of the palps and ovigers is similar. The basal element of the ovigers is here not considered an article, which is coincident with the currently established 10-articled (plus terminal claw) status. In addition, the palps of Colossendeis species should be considered as being 9-articled (8-articled in C. willsoni). Palp and oviger articulation conditions in Colossendeis show the most plesiomorphic conditions among the extant
Pycnogonida. The establishment of a similar basal structure in palps and ovigers could help in the evolutionary interpretation of these structures in general discussions on the evolution of Chelicerata, and in particular on the basal Pycnogonida groupings.

\section{Additional file}

Additional file 1: SM 1. External view showing basal parts of palps and ovigers. a, Dodecolopoda mawsoni. b, Decolopoda australis. Abbreviations: o1-04, first to fourth oviger article;obp, oviger's basal process; p1, first palp article; p2, second palp article; pbp, palp's basal process; pr, proboscis; tr, trunk.

\section{Abbreviations}

c1, c2, and c3: coxa 1, coxa 2 and coxa 3; emb: extrinsic muscle bands; imb: intrinsic muscle bands; I $p$ : lateral process on the trunk; $01,02,03, \ldots .:$ first, second, third, ... oviger articles; obp: oviger's basal process; $p 1, p 2, p 3, \ldots$. first, second, third, ... palp articles; pbp: palp's basal process. 


\section{Authors' contributions}

ECS conceived of the study, coordinated, drafted the manuscript, and carried out MO study. PJLG participated in the design of the study and its discussion, and carried out the SEM study. Both authors read and approved the final manuscript.

\section{Acknowledgements}

The authors would like to thank the officers and crew and many colleagues for their help on board during the VLT-2004 (Italica, XIX Spedizione) and the Polarstern ANT XXIII/8 cruises. We take this opportunity to extend our thanks to the cruise leader and steering committee of both cruises, especially Riccardo Cattaneo, Maria Chiara Chiantore and Roberto Meloni (VTL-2004), as well as Julian Gutt and Enrique Isla (ANT XIII/8), all of whom kindly facilitated the work on board and allowed us to collaborate in these Antarctic programmes. Partial support for this work was provided by the Spanish CICYT projects CGL200420062-E (Victoria Land Transect-2004) and POL2006-06399/CGL (Polarstern ANT XXIII/8 - CLIMANT). The authors thank three anonymous reviewers for their helpful corrections and suggestions. Mr. Tony Krupa is thanked for reviewing the English version.

\section{Competing interests}

The authors declare that they have no competing interests.

\section{Availability of data and material}

There are no data or examined material in this research which need special care or protection.

\section{Consent for publication}

All authors read and approved the final manuscript for publication in HMR.

\section{Ethics approval and consent to participants}

All authors agree that there are not ethics questions concerning the presented research.

\section{Funding}

Partial support (travel expenses for participation in polar cruises) was provided by the Spanish CICYT projects CGL2004-20062-E (Victoria Land Transect-2004) and POL2006-06399/CGL (Polarstern ANT XXIII/8_CLIMANT).

Received: 26 April 2016 Accepted: 11 October 2016

Published online: 15 December 2016

\section{References}

1. Boudreaux HB. Arthropod phylogeny with special reference to insects. New York: Wiley; 1979

2. Arango CP, Wheeler WC. Phylogeny of the sea spiders (Arthropoda, Pycnogonida) based on direct optimization of six loci and morphology. Cladistics. 2007:23:1-39.

3. Munilla T, Soler-Membrives A. Check-list of the pycnogonids from Antarctic and sub-Antarctic waters: zoogeographic implications. Antarct Sci. 2009:21:99-111.

4. Bamber RN. A holistic re-interpretation of the phylogeny of the Pycnogonida Latreille, 1810 (Arthropoda). Zootaxa. 2007;1668:295-312.

5. Arabi J, Cruaud C, Couloux A, Hassanin A. Studying sources of incongruence in arthropod molecular phylogenies: sea spiders (Pycnogonida) as a case study. C R Biol. 2010;333:438-53.

6. Arango CP. Morphological phylogenetics of the sea spiders (Arthropoda: Pycnogonida). Org Div Evol. 2002;2:107-25.

7. Dunlop JA, Arango CP. Pycnogonid affinities: a review. J Zool Syst Evol Res. 2005;43:8-21.

8. Arnaud F, Bamber R. The biology of Pycnogonida. Adv Mar Biol. 1987:24:1-96
9. Hedgpeth JW. On the evolutionary significance of the Pycnogonida. Smithson Misc Coll. 1947:106:1-54.

10. Munilla T. Evolución y Filogenia de los Picnogónidos. Bol Soc Entomol Aragonesa. 1999;26:273-9.

11. Stock JH. Indo-west pacific Pycnogonida collected by some major oceanographic expeditions. Beaufortia. 1994;1:17-77.

12. Child CA. Antarctic and Subantarctic Pycnogonida IV. The Family Colosendeidae and Rhynchothoraxidae. In: Cairns SD, editor. Biology of the Antarctic Seas XXIII. Antarct Res Ser. 1995;69:69-111.

13. Bouvier EL. Pycnogonides du Pourquoi Pas? Deux Expéd Antarc française (1908-1910). 1913;6:1-169.

14. Calman WT. Pycnogonida. British Antarctic (Terra Nova) expedition 1910. Zoology. 1915;3:1-74.

15. Gordon I. Pycnogonida. Disc Rep. 1932;6:1-138.

16. Gordon I. Pycnogonida. Rep Australas Antarct Exped C (Zoology and Botany). 1938;2:1-40

17. Gordon I. Pycnogonida. Rep Br Aust N Z Exp B. 1944;5:1-172.

18. Fry WG, Hedgpeth JW. Pycnogonida, 1. Colossendeidae, Pycnogonidae, Endeidae, Ammotheidae. Fauna of the Ross Sea 7. N Z Oceanogr Inst Mem. 1969:49:1-139.

19. Hoek PPC. Report on the Pycnogonida dredged by HMS Challenger 1873-1876. Rep Sci Res Challenger. 1881;3:1-167.

20. Wilson CB. Report on the Pycnogonida. Reports on the results of dredging, under the supervision of Alexander Agassiz, along the east coast of the United States, during the summer of 1880, by the US Coast Survey Steamer "Blake". Bull Mus Comp Zool Harvard Univ. 1881;8:239-56.

21. Hodgson TV. Pycnogonida. National Antarctic Expedition 1901-1904. Reports of the National Antarctic expedition of 1901-1904. Nat Hist. 1907:3:1-172.

22. Cano E, López-González PJ. Colossendeis species (Pycnogonida: Colossendeidae) collected during the Italica XIX cruise to Victoria land (Antarctica), with remarks on some taxonomic characters of the ovigers. Sci Mar. 2007:71:661-81.

23. Hodgson TV. The Pycnogonida collected by "Gauss" in the Antarctic Regions, 1901-1903. Prelim Rep Ann Mag Nat Hist. 1915;15:141-9.

24. Hodgson TV. Die pycnogoniden der deutschen südpolar-expedition 1901-1903. Zool. 1927;19:303-58.

25. Pushkin AF. The pycnogonida fauna of the South Ocean. Biological results of the soviet Antarctic expeditions. Russian Acad Sci. 1993;20:1-397.

26. Johansen DA. Plant microtechniques. New York: McGraw-Hill; 1940.

27. Arango CP. Molecular approach to the phylogenetics of sea spiders (Pycnogonida, Arthropoda) using nuclear ribosomal DNA and morphology. Mol Phylogenet Evol. 2003;28:588-600.

28. Bergström J, Sturmer W, Winter G. Palaeoisopus, Palaeopantopus and Palaeothea,pycnogonid arthropods from the Lower Devonian Hunsrück Slate, West Germany. Paläont Z. 1980;54:7-54.

29. Denker VD. Das Skeletmuskelsystem von Nymphon rubrum Hodge, 1862 (Pycnogonida: Nymphonidae). Zool Jb Anat. 1974;93:272-87.

30. Schram FR, Hedgpeth JW. Locomotory mechanisms in Antarctic pycnogonids. Zool J Linn Soc-Lond. 1978:63:145-69.

\section{Submit your next manuscript to BioMed Central and we will help you at every step:}

- We accept pre-submission inquiries

- Our selector tool helps you to find the most relevant journal

- We provide round the clock customer support

- Convenient online submission

- Thorough peer review

- Inclusion in PubMed and all major indexing services

- Maximum visibility for your research

Submit your manuscript at www.biomedcentral com/submit 